\title{
Application of tablet for data collection in HIV sentinel surveillance in Vietnam
}

\author{
Duong C. Thanh"1, Ha T. Nguyen', Giang T. Le², Duc H. Bui ${ }^{3}$, Lo T. Dang ${ }^{3}$, Diep T. Vu², \\ Nghia V. Khuu ${ }^{4}$, Tuan A. Nguyen ${ }^{1}$ and Huong T. Phan ${ }^{3}$
}

${ }^{1}$ HIV/AIDS, National Institute of Hygiene and Epidemiology, Ha Noi, Viet Nam; ${ }^{2}$ Centers for Disease Control and Prevention, Hanoi, Viet Nam; ${ }^{3}$ Vietnam Authority of HIV/AID Control, Hanoi, Viet Nam; ${ }^{4}$ Pasteur Institute in Ho Chi Minh City, Ho Chi Minh, Viet Nam

\section{Objective}

To describe the implementation process, successes, challenges, and lessons learned of the application of tablet for data collection and data system in HIV sentinel surveillance in Vietnam

\section{Introduction}

Vietnam has routinely monitored HIV sero-prevalence among key populations through its HIV sentinel surveillance system (HSS). In 2010, this system was updated to include a behavioral component (HSS+) among people who inject drugs, female sex workers, and men who have sex with men. HSS+ has historically used a paperbased questionnaire for data collection ${ }^{(1)}$. At the end of the survey, provincial data were manually entered into computers using EpiData Entry forms (http://www.epidata.dk/) and submitted to the Vietnam Authority of HIV/AIDS Control (VAAC). As a result, feedback to provinces on data issues was not provided until after fieldwork completion. One recent survey used tablets for data collection and found that it saved time, required fewer staff, and reduced costs compared to paper-based data collection ${ }^{(2)}$. In 2017, Vietnam introduced tablet for behavioral data collection in HSS + to improve data quality, resource saving, and to provide more timely access to data.

\section{Methods}

Development of data entry forms and data system

Survey data entry forms were designed using free Epi Info ${ }^{\mathrm{TM}}$ software for mobile devices ${ }^{(3)}$ and installed on tablets. A SQL database was established via SFPT data transfer to the current database in VAAC's server. Field data were instantly synced to the national database when the internet signal was available (Picture 1). Real-time data analysis was granted to surveillance staff at all levels using authorized access to the database via Epi Info ${ }^{\text {TM }}$ Cloud Data Analytics (ECDA), dashboards were used to track progress and data quality (Figure 1). HSS + data were frequently reviewed by the National Surveillance Technical Working Group (NSTWG) and timely feedback was provided.

Deployment

Manuals and e-leaning materials were developed. The NSTWG conducted a pilot to test the forms and data flow from field to the national database before installed into all tablets. Four to seven tablets were distributed to each province depending on number of HSS + sites and populations. Surveillance staff at Provincial AIDS Centers (PACs) were trained by the NSTWG on how to use the tablet to interview, check, update, save data, and sync data to cloud and to the national database, and to backup the provincial dataset. They then provided trainings to their local field staff.

The NSTWG provided technical assistance and troubleshooting through field visits and online support to help local staff address issues regarding tablet use in addition to other HSS/HSS+ issues.

\section{Results}

Currently, 18 HSS+ provinces have implemented the 2017 HSS+. Of these, nine provinces applied tablets exclusively. Two provinces used tablets, but also used paper-based questionnaires when not enough tablets were available. Seven Global Fund supported provinces used the paper-based questionnaires and entered data into tablets after interview completion due to copies of completed paper-based questionnaires are required by these provincial project management units (PMU) for fund re-imbursement.

Additional updates were required after the first few days, which created issues around updating forms once revised forms were sent out by NSTWG. Another challenge was that local staff were not familiar with using tablets at the beginning. Also frequent complaints were mainly on data entry and synchronization regarding participant identity code or a record could not be synced.

The NSTWG and PAC staff were able to monitor the HSS+ progress and provided feedback daily. Most commonly, feedbacks were provided on participant codings and site names. Using the tablet did not require staff, time or money for data entry and eliminated data entry errors. In general, staff prefered to use this data collection mode.

\section{Conclusions}

This mobile device application for data collection in routine HSS+ in Vietnam is feasible and accepted. However, harmonization and coordination from the central Global Fund PMU and provincial PMU will be required to successfully roll-out this system in all HSS+ provinces. This application in addition to ECDA help to improve data quality, due to timeliness of the data, is cost saving and reduces workload. Most importantly, better quality and timely data will facilitate preparation for timely local planning and response.

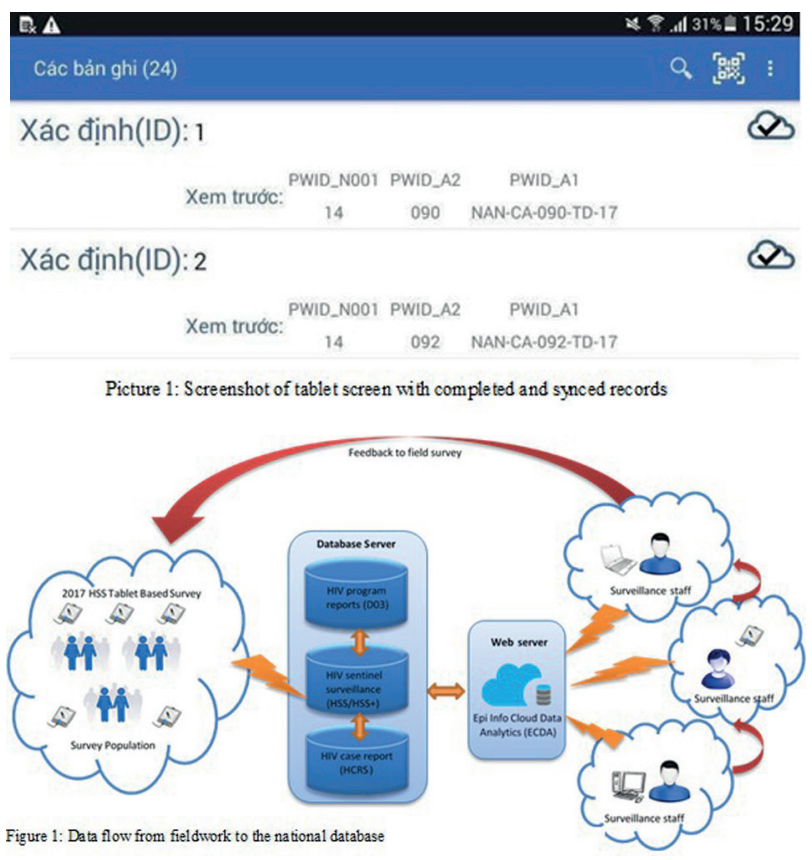




\section{ISDS 2018 Conference Abstracts}

Keywords

HSS; HSS+; tablet; mobile device; data collection

\section{Acknowledgments}

To the survey participants, surveillance staff at all level for their dedicated work on the HSS/HSS+, and the US CDC for ECDA deployment and tablets through PEPFAR program.

\section{References}

1. Thanh DC et al. Brief behavioural surveys in routine HIV sentinel surveillance: a new tool for monitoring the HIV epidemic in Vietnam.

Western Pacific Surveillance and Response Journal. Vol 6, No. 1/2015

2. National Institute of Hygiene and Epidemiology. HIV/STI Integrated Biological and Behavioural Surveillance in Vietnam. Hanoi, 2014.

3. https://www.cdc.gov/epiinfo/mobile.html

\section{*Duong C. Thanh}

E-mail: congthanhnihe@yahoo.com 